\title{
luxS Mutant Regulation: Quorum Sensing Impairment or Methylation Disorder?
}

\author{
Qian Wang ${ }^{1, \dagger}$, Zhiyan He ${ }^{1, \dagger}$,Yuejian Hu ${ }^{1,2}$, Yuntao Jiang ${ }^{1}$, Rui Ma ${ }^{1}$, Zisheng Tang ${ }^{1}$, \\ Jingping Liang ${ }^{1, *}$, Zheng Liu ${ }^{1}$ and Zhengwei Huang ${ }^{1, *}$
}

1 Department of Endodontics, Ninth People's Hospital, Shanghai Key Laboratory of Stomatology, School of Medicine, Shanghai Jiao Tong University, No. 639 Zhizaoju Road, Shanghai 200011, China; E-Mails: qtdc1010@sjtu.edu.cn (Q.W.); zyhe23@126.com (Z.H.); hyjian@sjtu.edu.cn (Y.H.); jiangyuntao1974@163.com (Y.J.); marui6868@yahoo.com (R.M.); tangzisheng@yahoo.com.cn (Z.T.); sris@sh163.net (Z.L.)

2 Department of Endodontics, The Affiliated Stomatology Hospital of Tongji University, School of Stomatology, Tongji University, Shanghai 200072, China

$\dagger$ These authors contributed equally to this work.

* Authors to whom correspondence should be addressed; E-Mails: liangjp123@yahoo.com.cn (J.L.); huangzhengwei@shsmu.edu.cn (Z.H.); Tel./Fax:+86-21-6313-5412.

Received: 19 March 2012; in revised form: 12 April 2012 / Accepted: 20 April 2012 /

Published: 10 May 2012

\begin{abstract}
AI-2-mediated quorum sensing has been identified in various bacteria, including both Gram-negative and Gram-positive species, and numerous phenotypes have been reported to be regulated by this mechanism, using the luxS-mutant strain. But the AI-2 production process confused this regulatory function; some considered this regulation as the result of a metabolic change, which refers to an important metabolic cycle named activated methyl cycle (AMC), caused by luxS-mutant simultaneously with the defect of AI-2. Herein we hypothesized that the quorum sensing system-not the metabolic aspect-is responsible for such a regulatory function. In this study, we constructed plasmids infused with $s a h H$ and induced protein expression in the luxS-mutant strain to make the quorum-sensing system and metabolic system independent. The biofilm-related genes were investigated by real-time polymerase chain reaction (PCR), and the results demonstrated that the quorum-sensing completed strain restored the gene expression of the defective strain, but the metabolically completed one did not. This evidence supported our
\end{abstract}


hypothesis that the autoinducer-2-mediated, quorum-sensing system, not the AMC, was responsible for luxS mutant regulation.

Keywords: quorum sensing; AI-2; luxS; sahH

\section{Introduction}

Quorum sensing (QS) is an intercellular signal mechanism that bacteria use to control their gene expression for adapting to changes in their surroundings. This process involves the production, secretion, and recognition of signal molecules and regulation of gene expression [1]. Among these signal molecules, autoinducer-2 (AI-2), which was first identified as a regulator of bioluminescence in Vibrio harveyi [2], supposedly participates in interspecies cell-to-cell communication because of its generating gene luxS that conservatively exists in a broad range of species [3].

Previous studies have used the $\operatorname{luxS}$ mutant strain to demonstrate that AI-2-mediated QS plays an important regulative role in many biological behaviours of bacteria such as cell division, DNA processing, virulence, and motility [4-8]. Furthermore, as an important etiological factor of infectious diseases [9-11], biofilm formation also differs between luxS mutant and its wild-type strain [12-15]. There is much controversy over the origin of these regulating behaviors. AI-2 is a byproduct of the activated methyl cycle (AMC) [16] (Figure 1). This important metabolic cycle begins with homocysteine (HCY), then involves the formation of methione (MET), $S$-adenosylmethionine (SAM), and $S$-adenosylhomocysteine (SAH). During the cycle, SAM provides an activated methyl group $\left(\mathrm{CH}_{3}\right)$, which is used for the methylation of RNA, DNA, certain metabolites, and proteins [17,18]. To complete the cycle, HCY is generated from SAH through two independent pathways. Some bacteria, like Escherichia coli, travel one pathway that needs Pfs enzyme to first produce $S$-ribosylhomocysteine (SRH), then LuxS catalyzes the conversion from SRH to HCY, simultaneously generating the precursor of AI-2, 4,5-dihydroxy-2,3-pentanedione (DPD). The other route involves a one-step conversion catalyzed by SAH hydrolase $(\mathrm{SahH})$, which catalyzes the conversion from SAH to adenosine and HCY but without generating DPDs [19-21], bacteria such as P. aeruginosa which do not contain luxS passing through this way. Since most previous studies on mutated $\operatorname{lux} S$ considered it a QS-defective strain and that AMC was also disrupted at the LuxS level, a series change would occur in the bacteria's metabolism [20]. Thus arose the controversy as to whether the change in gene expression is caused by QS, as previously described, or by AMC metabolic pathway disruption [21,22].

Although the luxS mutant leads to AMC obstruction, it is not believed to arouse so much regulated gene expression belonging to such diverse biological behaviors as gene production, virulence, and motility [7,23]. Such a wide influence is likely to be due to a monolithic mechanism, such as AI-2-induced QS. Furthermore, in previous studies, mutated luxS was not fatal to the strain, so we speculated that the influential range of the AMC obstacle was limited. To this end, we hypothesized that the QS role, instead of an AMC obstacle, is responsible for luxS mutant regulation. To verify our hypothesis, we intended to express $\mathrm{SahH}$ in an E. coli luxS mutant strain, aiming to repair the AMC of this strain but retain the defective QS system, then detect whether the genes reported to be regulated by AI-2-mediated QS would be restored. 
Figure 1. Process of activated methyl cycle (AMC; adapted from Vendeville et al. [18]). $E$. coli uses a two-step mechanism involving the Pfs and LuxS enzymes to produce the AI-2 precursor and homocysteine (HCY), while P. aeruginosa synthesizes HCY from S-adenosylhomocysteine (SAH) in a one-step reaction involving the SahH enzyme.

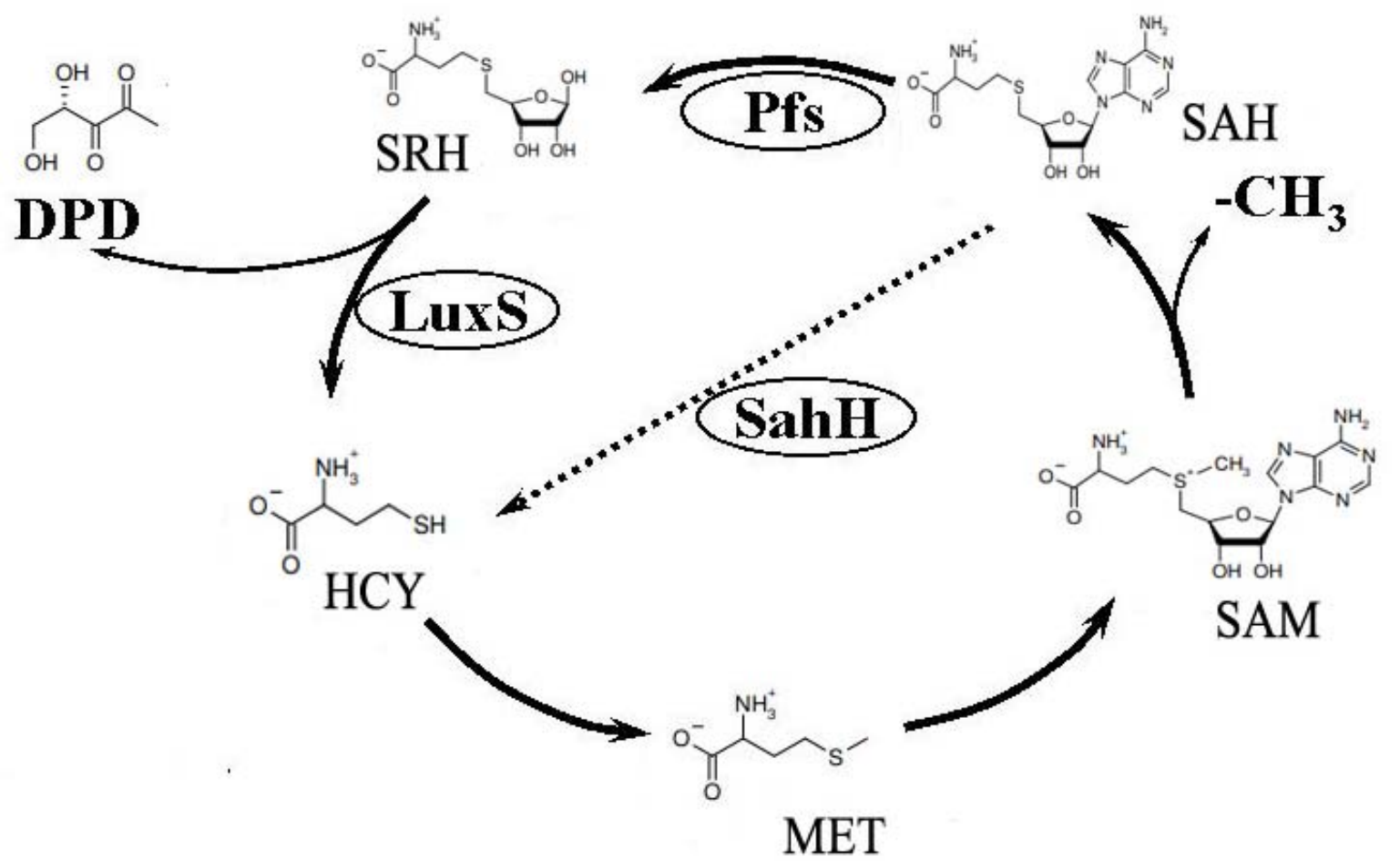

\section{Materials and Methods}

\subsection{Bacterial Strains, Plasmid Construction, and Culture Condition}

The strains and plasmids used in this study are listed in Table 1, the primers in Table 2. The sahH gene was amplified from genomic DNA of the P. aeruginosa strain PAO1 using primers SF and SR, and the $\operatorname{luxS}$ gene was amplified from genomic DNA of E. coli W3110 with primers LF and LR. The restriction enzymes chosen in this part were BamH I and EcoR I for sahH and vector, EcoR I and $\mathrm{Bgl}$ II for $\operatorname{luxS}$. And Bgl II is the isocaudamer of BamH I. To construct the plasmids pluxS and psahH, amplified products were inserted into the expression vector pGEX4T-1, using the standard method of preparing clones [24]. Generally, DNA amplification products were digested by the corresponding restriction enzymes and ligated with the vector. After sequencing, pluxS, psahH, and pGEX4T-1 were transformed into strain MDAI2 (luxS mutant strain) to construct strains M-L, M-S, and M-P, respectively.

All strains were precultured in a $2 \times$ YT medium at $37{ }^{\circ} \mathrm{C}$ overnight with shaking at $220 \mathrm{rpm}$, and the concentration of ampicillin for selection was $100 \mu \mathrm{g} / \mathrm{mL}$. These overnight cultures were inoculated into a fresh medium and cultivated at $37^{\circ} \mathrm{C}$ with 250 -rpm shaking. When the strains had reached the exponential phase, a final concentration of $0.1 \mathrm{mmol} / \mathrm{L}$ isopropyl- $\beta$-D-thiogalactopyranoside (IPTG) was added, and cultivation was continued for about $6 \mathrm{~h}$. 
Table 1. Plasmids and strains used in this study.

\begin{tabular}{lll}
\hline $\begin{array}{l}\text { Strain or } \\
\text { plasmid } \\
\text { Plasmids }\end{array}$ & Relevant genotype & Reference or source \\
\hline pGEX4T-1 & expression vector, Ap ${ }^{\mathrm{r}}$ & $\begin{array}{l}\text { Shanghai key laboratory } \\
\text { of stomatology } \\
\text { this study }\end{array}$ \\
pluxS & luxS from . coli W3110 cloned in pGEX4T-1 & this study \\
psahH & sahH from P. aeruginosa PAO1 cloned in pGEX4T-1 & \\
Strains & & \\
E.coli & & {$[25]$} \\
W3110 & K-12 strain, wild type & {$[25]$} \\
MDAI2 & W3110 luxS::TcrW3110-derived luxS mutant strain & this study \\
M-L & MDAI2 pluxS & $\begin{array}{l}\text { this study } \\
\text { this study }\end{array}$ \\
M-S & MDAI2 psahH & Shanghai key laboratory \\
M-P & MDAI2 pGEX4T-1 & of stomatology \\
P. aeruginosa & & \\
PAO1 & wild type & \\
\hline
\end{tabular}

Table 2. Primers used in this study.

\begin{tabular}{ll}
\hline Primer & Sequence (5'-3') \\
\hline SF & GGCGGATCCATGAGCGCTGTCATGACGC * \\
SR & GGCGAATTCTTAGTAGCGATAGGTGTCCGG \\
LF & GGCAGATCTATGCCGTTGTTAGATAGCTTCAC \\
LR & GGCGAATTCCTAGATGTGCAGTTCCTGCAACT \\
fliAF & CCGCAACGCCACGGAAACTGA \\
fliAR & GCTCTTCGCGCCACTCATCGTA \\
flicF & ATTGCTAACCGTTTCACCTCTAA \\
flicR & CGCTGTAAGTTGTTGTTGATTTCG \\
motAF & CGTCGCTCCAAATACACCAA \\
motAR & CAGCGAAAACATCCCCATCT \\
motBF & GCCAGCGGTGAGAAAGGA \\
motBR & CAACCCTCCGACCATCAGTT \\
rpoAF & GCGCTCATCTTCTTCCGAAT \\
rpoAR & CGCGGTCGTGGTTATGTG \\
\hline
\end{tabular}

\subsection{Western Blotting}

After cultivation, the $\mathrm{OD}_{600}$ of five strains was tested and total proteins were extracted. Briefly, about $2 \times 10^{8} \mathrm{E}$. coli were collected and a corresponding volume of double-distilled water $\left(\mathrm{ddH}_{2} \mathrm{O}\right)$ and a $5 \times$ sample of buffer (20\% sodium dodecyl sulfate [SDS], 20\% glycerol, $200 \mathrm{mM}$ Tris base, $\mathrm{pH} 6.8$, $0.001 \%$ bromophenol blue) was used to subsequently suspend the pellets. The samples were heated at $100{ }^{\circ} \mathrm{C}$ for $10 \mathrm{~min}$ and centrifuged for $1 \mathrm{~min}$. Equal amounts of total proteins were electrophoresed in SDS-10\% polyacrylamide gel electrophoresis. Western blotting was performed as previously 
described [24]. The antibodies used in this study were monoclonal anti-glutathione-S-transferase (anti-GST) and rabbit antimouse antibody with a green fluorescent group.

\subsection{RNA Extraction and Reverse Transcription}

After IPTG induction and cultivation for $6 \mathrm{~h}$, the total RNA of these strains was extracted. Culture volumes equivalent to $10 \mathrm{~mL}$ with an $\mathrm{OD}_{600}$ of 1.0 were harvested by centrifugation. The pellet was suspended with TRIzol (Invitrogen, Carlsbad, CA, USA). The reagent and RNA extraction process followed the manufacturer's specifications. The resulting RNA was dissolved in diethyl pyrocarbonate-treated water and stored at $-80{ }^{\circ} \mathrm{C}$. The PrimerScript gDNA eraser RT reagent kit (Takara, Otsu, Shiga, Japan) was used to generate cDNA; about $1 \mu \mathrm{g}$ of total RNA was used for each strain. To detect the target exogenous gene, cDNA of each strain was used as a template for polymerase chain reaction (PCR). Other compositions were forward/reverse primer (SF, SR, LF, LR), PrimeStar HS DNA Polymerase (Takara) and buffer, dNTP mixture, and $\mathrm{ddH}_{2} \mathrm{O}$. PCR conditions included an initial denaturation at $98^{\circ} \mathrm{C}$ for $5 \mathrm{~min}$, followed by 30 cycles amplification consisting of $98^{\circ} \mathrm{C}$ for $15 \mathrm{~s}$, $55{ }^{\circ} \mathrm{C}$ for $15 \mathrm{~s}$, and $72{ }^{\circ} \mathrm{C}$ for $90 \mathrm{~s}$, then final extension at $72{ }^{\circ} \mathrm{C}$ for $5 \mathrm{~min}$. The amplified products were electrophoresed in $1 \%$ agarose gel.

\subsection{Real-Time PCR}

To determinae whether the AMC-completed strain would restore the biofilm formation-related genes affected by $\operatorname{luxS}$ mutant, the expression of these genes was compared with the real-time PCR. The target genes and their primers are shown in Table 2. The amplification efficiency and template specificity of each primer pair were verified, then the amplification was performed with the following 15- $\mu \mathrm{L}$ reaction mixture: $7.5 \mu \mathrm{L} 2 \times$ Thunderbird SYBRqPCR Mix (Toyobo, Osaka, Japan), $5 \mu \mathrm{L}$ cDNA

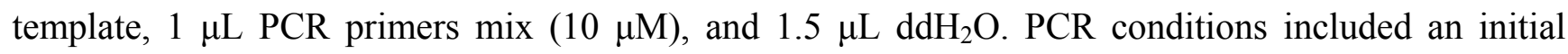
denaturation at $98^{\circ} \mathrm{C}$ for $5 \mathrm{~min}$, followed by 40 cycles amplification of $98{ }^{\circ} \mathrm{C}$ for $15 \mathrm{~s}, 55^{\circ} \mathrm{C}$ for $15 \mathrm{~s}$, and $72{ }^{\circ} \mathrm{C}$ for $30 \mathrm{~s}$. To check DNA contamination, the production of RT-PCR step 1 (without reverse transcriptase) served as a negative control. The rpoA gene was used in this study as the normalising gene for all reactions [26]. Applied Biosystems (Carlsbad, CA, USA) 7900HT Fast Real Time PCR System was used for this test, and fold changes of the expression levels were calculated by SDS Software v2.3 with RQ Study 1.2 (Applied Biosystems). All the assays were conducted with each sample in triplicate. Here, the Kruskal-Wallis test was used to analyse the gene expression difference among the five strains. A $P$-value less than 0.05 was considered statistically significant.

\section{Results and Discussion}

\subsection{Identification of Exogenous Gene Expression}

To test and verify our hypothesis, the QS system and AMC must be independent. Previous investigators have collected supernatant that had cultivated a wild-type strain for some time, believing it to contain AI-2 [27,28]. They then used it as a conditioning medium in which to cultivate the luxS mutant strain. In a sense, this method could make the QS system and AMC independent, but it allowed the other substance in the supernatant - secreted by the bacteria - to influence the results. This study, 
aiming to restore the obstructed AMC and simultaneously keep the defective QS mechanism, transformed the sahH into an E. coli luxS mutant strain. Consequently, we could now check whether the AMC or the QS system took responsibility for changes caused by the luxS mutant.

The RT-PCR and Western blot were performed to prove the expression of target genes in these five strains. As shown in Figure 2, the bands detectable by anti-GST represented the fusion proteins GST-LuxS and GST-SahH, whose molecular weights were about $44.9 \mathrm{kDa}$ and $77.7 \mathrm{kDa}$, respectively. For $\operatorname{luxS}$ RT-PCR, the primer pairs LF and LR could spot both W3110 and M-L, whereas sahH was detected only in M-S. Moreover, the band amplified from M-L is more remarkable than W3110, which amplification should be attributed to the overexpression of pluxS.

Figure 2. GST-tagged LuxS and SahH were detected in strains M-L and M-S, respectively, by Western blotting. RT-PCR showed that $\operatorname{luxS}$ existed in both W3110 and M-L, while M-L is more likely caused by gene overexpression. $s a h H$ was detectable only in M-S.

\section{W3110 MDAI2 M-L M-S M-P}

GST-LuxS

GST-SahH
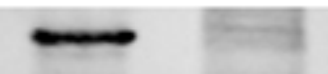

. 
showed that there was no significant restoration by expression of $\mathrm{SahH}$ which was intended to complete the AMC, thus the luxS-mediated QS system impacted the biofilm-related gene expression.

This study also showed that, the genes, downregulated by the luxS mutant, were even strongly upregulated when LuxS was overexpressed. This demonstrated that the mutant strain with LuxS expression may complete the QS system and cause AI-2 secretion to recover the expression of the target genes. And theoretically, overexpressed LuxS synthetized more active AI-2, so these target genes were upregultaed much more than the wild-type strain. But under the same experimental conditions, the mutant strain with SahH expression, which might recover the AMC system without AI-2 secretion, kept the expression of target genes at a low level (Figure 3), which means that, without integrated QS mediating, the phenotype could not be recovered, although its role in metabolism may be intact.

Figure 3. Real-time PCR was used to demonstrate that motility-related genes are restored in the luxS-mutant strain by the expression of $\operatorname{lux} S$, although it was not restored in the mutant strain with the plasmid-expressing sahH. The results represent the means and standard deviations (SDs) of relative quantification.
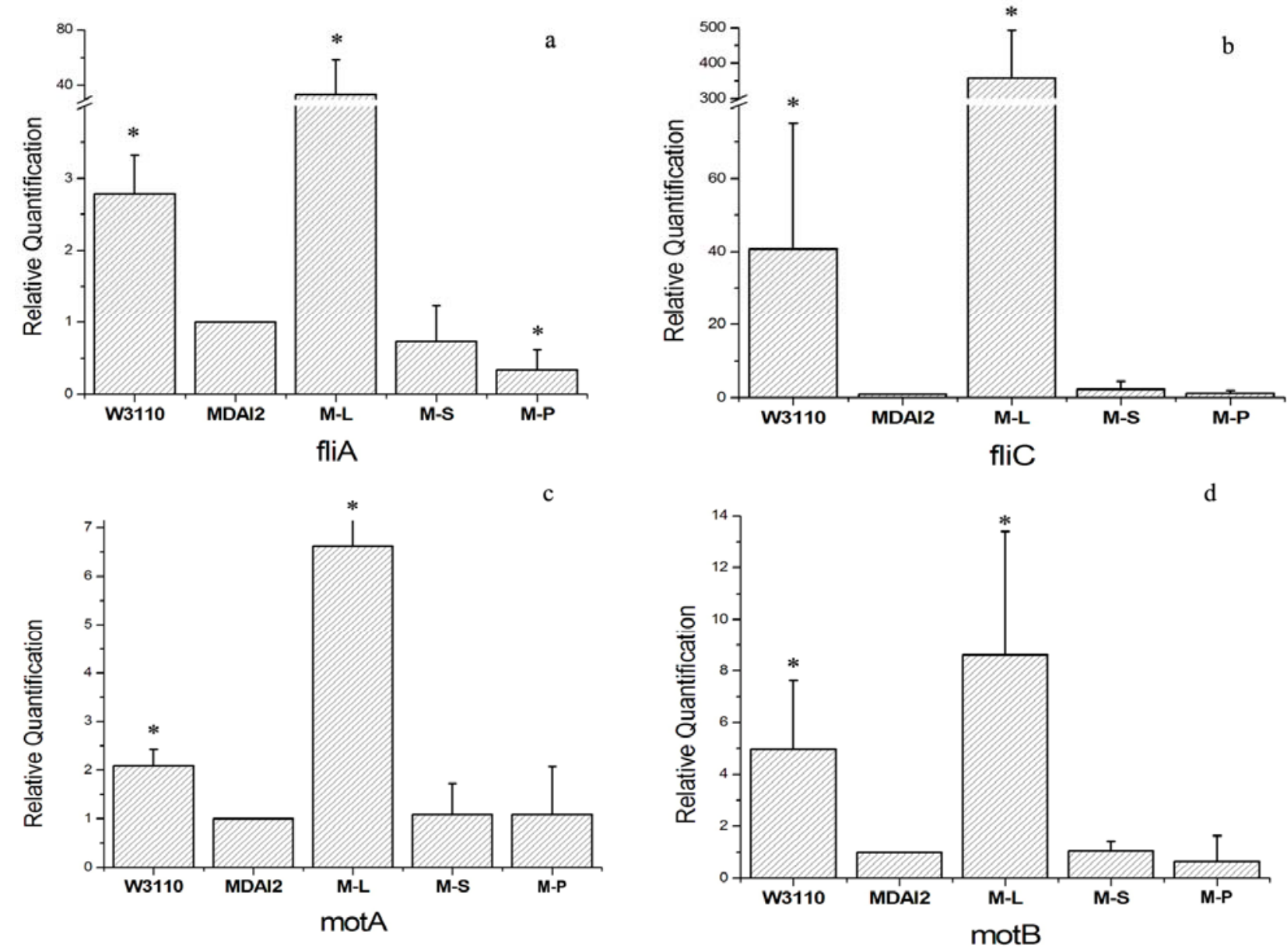

* Statistically significant differences $(P<0.05)$ compared with MDAI2. 


\section{Conclusions}

The genetic test results confirm our hypothesis that the QS role-rather than metabolism - is the primary regulator of $l u x S$ mutant physiological changes. We intend, in a future study, to further verify our hypothesis by means of an E.coli function test.

\section{Acknowledgments}

We thank William E. Bentley of the University of Maryland Biotechnology Institute for the W3110 and MDAI2 strains used in this work. This study was supported by a grant from the National Natural Science Foundation of China (NSFC) No. 81070826/30872886, and partly supported by a university grant of YG2011MS67.

\section{References}

1. Xavier, K.B.; Bassler, B.L. Regulation of Uptake and Processing of the Quorum-Sensing Autoinducer AI-2 in Escherichia coli. J. Bacteriol. 2005, 187, 238-248.

2. Bassler, B.L.; Wright, M.; Showalter, R.E.; Silverman, M.R. Intercellular Signalling in Vibrio Harveyi: Sequence and Function of Genesregulating Expression of Luminescence. Mol. Microbiol. 1993, 9, 773-786.

3. Xavier, K.B.; Bassler, B.L. luxS Quorum Sensing: More than just a Numbers Game. Curr. Opin. Microbiol. 2003, 6, 191-197.

4. Walters, M.; Sircili, M.P.; Sperandio, V. AI-3 Synthesis is not Dependent on luxS in Escherichia coli. J. Bacteriol. 2006, 188, 5668-5681.

5. Li, J.; Attila, C.; Wang, L.; Wood, T.K.; Valdes, J.J.; Bentley, W.E. Quorum Sensing in Escherichia coli is Signaled by AI-2/LsrR Effects on Small RNA and Biofilm Architecture. J. Bacteriol. 2007, 189, 6011-6020.

6. Ling, H.; Kang, A.; Tan, M.H.; Qi, X.; Chang, M.W. The Absence of the luxS Gene Increases Swimming Motility and flagella Synthesis in Escherichia coli K12. Biochem. Biophys. Res. Commun. 2010, 401, 521-526.

7. Delisa, M.P.; Wu, C.P.; Wang, L.; Valdes, J.J.; Bentley, W.E. DNA Microarray-Based Identification of Genes Controlled by Autoinducer 2-Stimulated Quorum Sensing in Escherichia coli. J. Bacteriol. 2001, 183, 5239-5247.

8. DeLisa, M.P.; Valdes, J.J.; Bentley, W.E. Mapping Stress-Induced Changes in Autoinducer AI-2 Production in Chemostat-Cultivated Escherichia coli K-12. J. Bacteriol. 2001, 183, 2918-2928.

9. Huang, Z.W.; Meric, G.; Liu, Z.; Ma, R.; Tang, Z.S.; Lejeune, P. luxS-Based Quorum-Sensing Signaling Affects Biofilm Formation in Streptococcus mutans. J. Mol. Microbiol. Biotechnol. 2009, 17, 12-19.

10. Ma, R.; Liu, J.; Jiang, Y.T.; Liu, Z.; Tang, Z.S.; Ye, D.X.; Zeng, J.; Huang, Z.W. Modeling of Diffusion Transport through Oral Biofilms with the Inverse Problem Method. Int. J. Oral Sci. 2010, 2, 190-197.

11. Huang, Z.W.; Jiang, Y.T.; Liang, J.P. Pathogenesis could be One of the Anti-Cheating Mechanisms for Pseudomonas aeruginosa Society. Med. Hypoth. 2011, 76, 166-168. 
12. Balestrino, D.; Haagensen, J.A.J.; Chantal, R.; Forestier, C. Characterization of Type 2 Quorum Sensing in Klebsiella pneumoniae and Relationship with Biofilm Formation. J. Bacteriol. 2005, 187, 2870-2880.

13. Lee, J.; Maeda, T.; Hong, S.T.; Wood T.K. Reconfiguring the Quorum-Sensing Regulator SdiA of Escherichia coli to Control Biofilm Formation via Indole and N-Acylhomoserine Lactones. Appl. Microbiol. Biotechnol. 2009, 75, 1703-1716.

14. Ren, D.; Bedzyk, L.; Ye, R.W.; Thomas, S.; Wood, T.K. Differential Gene Expression Shows Natural Brominated Furanones Interfere with the Autoinducer-2 Bacterial Signaling System of Escherichia coli. Biotechnol. Bioeng. 2004, 88, 630-642.

15. Sperandio, V.; Torres, A.G.; Giron, J.A.; and Kaper, J.B. Quorum Sensing is a Global Regulatory Mechanism in Enterohemorrhagic Escherichia coli O157:H7. J. Bacteriol. 2001, 183, 5187-5197.

16. Winzer, K.; Hardie, K.R.; Burgess, N.; Doherty, N.; Kirke, D.; Holden, M.T.G.; Linforth, R.; Cornell, K.A.; Taylor, A.J.; Hill, P.J.; Williams, P. luxS: Its Role in Central Metabolism and the in vitro Synthesis of 4-hydroxy-5-methyl-3(2H)-furanone. Microbiology 2002, 148, 909-922.

17. Winzer, K.; Hardie, K.R.; Williams, P. luxS and Autoinducer-2: Their Contribution to Quorum Sensing and Metabolism in Bacteria. Adv. Appl. Microbiol. 2003, 53, 291-396.

18. Vendeville, A.; Winzer, K.; Heurlier, K.; Tang, C.M.; Hardie, K.R. Making 'Sense' of Metabolism: Autoinducer 2, luxS and Pathogenic Bacteria. Nat. Rev. Microbiol. 2005, 3, 383-396.

19. Nikhat, P.; Kenneth, A.C. Methylthioadenosine/S-Adenosylhomocysteine Nucleosidase, a Critical Enzyme for Bacterial Metabolism. Mol Microbiol. 2011, 79, 7-20.

20. Halliday, N.M.; Hardie, K.R.; Williams, P.; Winzer, K.; Barrett, D.A. Quantitative Liquid Chromatography-Tandem Mass Spectrometry Profiling of Activated Methyl Cycle Metabolites Involved in luxS-Dependent Quorum Sensing in Escherichia coli. Anal. Biochem. 2010, 403, 20-29.

21. Sun, J.B.; Daniel, R.; Wagner-Döbler, I.; Zeng, A.-P. Is Autoinducer-2 a Universal Signal for Interspecies Communication: A Comparative Genomic and Phylogenetic Analysis of the Synthesis and Signal Transduction Pathways. BMC Evol. Biol. 2004, 4, doi: 10.1186/1471-21484-36.

22. Winzer, K.; Hardie, K.R.; Williams, P. Bacterial Cell-to-Cell Communication: Sorry, can't Talk Now-Gone to Lunch! Curr. Opin. Microbiol. 2002, 5, 216-222.

23. Wang, L.; Li, J.; March, J.C.; Valdes, J.J.; Bentley, W.E. luxS-Dependent Gene Regulation in Escherichia coli K-12 Reveale by Genomic Expression Profiling. J. Bacteriol. 2005, 187, 8350-8360.

24. Sperandio, V.; Li, C.C.; Kaper, J.B. Quorum-Sensing Escherichia coli Regulator A: A Regulator of the LysR Family Involved in the Regulation of the Locus of Enterocyte Effacement Pathogenicity Island in Enterohemorrhagic E. coli. Infect. Immun. 2002, 70, 3085-3093.

25. Tsao, C.Y.; Wang, L.; Hashimoto, Y.; Yi, H.; March, J.C.; DeLisa, M.P.; Wood, T.K.; Valdes, J.J.; Bentley, W.E. luxS Coexpression Enhances Yields of Recombinant Proteins in Escherichia coli in Part through Posttranscriptional Control of GroEL. Appl. Environ. Microbiol. 2011, 77, 2141-2152.

26. Melissa, M.K.; Rasko, D.A.; Sperandio, V. Global Effects of the Cell-to-Cell Signaling Molecules Autoinducer-2, Autoinducer-3, and Epinephrine in a luxS Mutant of Enterohemorrhagic Escherichia coli. Infect. Immun. 2007, 75, 4875-4884. 
27. Sperandio, V.; Torres, A.G.; Kaper, J.B. Quorum Sensing Escherichia coli Regulators B and C (QseBC): A Novel Two-Component Regulatory System Involved in the Regulation of fllagella and Motility by Quorum Sensing in E. coli. Mol. Microbiol. 2002, 43, 809-821.

28. Yoon, Y.; Sofos, J.N. Absence of Association of Autoinducer-2-Based Quorum Sensing with Heat and Acid Resistance of Salmonella. J. Food Sci. 2010, 75, M444-M448.

29. Pratt, L.A.; Kolter, R. Genetic Analysis of Escherichia coli Biofilm Formation: Roles of fllagella, Motility, Chemotaxis and Type I pili. Mol. Microbiol. 1998, 30, 285-293.

30. Habdas, B.J.; Smart, J.; Kaper, J.B.; Sperandio, V. The LysR-Type Transcriptional Regulator QseD Alters Type Three Secretion in Enterohemorrhagic Escherichia coli and Motility in K-12 Escherichia coli. J. Bacteriol. 2010, 192, 3699-3712.

31. Barrios, A.G.; Zuo, R.; Hashimoto, Y.; Yang, L.; Bentley, W.E.; Wood, T.K. Autoinducer 2 Controls Biofilm Formation in Escherichia coli through a Novel Motility Quorum-Sensing Regulator (MqsR, B3022). J. Bacteriol. 2006, 188, 305-316.

32. Domka, J.; Lee, J.; Bansal, T.; Wood, T.K. Temporal Gene-Expression in Escherichia coli K-12 Biofilms. Environ. Microbiol. 2007, 9, 332-346.

33. Blattner, F.R.; Plunkett, G.; Bloch, C.A.; Perna, N.T.; Burland, V.; Riley, M.; Collado-Vides, J.; Glasner, J.D.; Rode, C.K.; Mayhew, G.F.; Gregor, J.; Davis, N. W.; Kirkpatrick, H.A.; Goeden, M.A.; Rose, D.J.; Mau, B.; Shao, Y. The Complete Genome Sequence of Escherichia coli K-12. Science 1997, 277, 1453-1474.

34. Ren, D.; Bedzyk, L.; Thomas, S.; Ye, R.W.; Wood, T.K. Gene Expression in Escherichia coli Biofilms. Appl. Microbiol. Biotechnol. 2004, 64, 515-524.

(C) 2012 by the authors; licensee MDPI, Basel, Switzerland. This article is an open access article distributed under the terms and conditions of the Creative Commons Attribution license (http://creativecommons.org/licenses/by/3.0/). 